\title{
Correlation of Shape and Size of Sella Turcica With the Type of Facial Skeletal Class in an Iranian Group
}

\author{
Solmaz Valizadeh, ${ }^{1}$ Shahrzad Shahbeig, ${ }^{1}$ Sudeh Mohseni, ${ }^{2}$ Fateme Azimi, ${ }^{3, *}$ and Hooman \\ Bakhshandeh $^{4}$ \\ ${ }^{1}$ Department of Oral and Maxillofacial Radiology, School of Dentistry, Shahid Beheshti University of Medical Sciences, Tehran, Iran \\ ${ }_{2}^{2}$ Department of Oral and Maxillofacial Radiology, School of Dentistry, Kerman University of Medical Sciences, Kerman, Iran \\ ${ }^{3}$ Department of Oral and Maxillofacial Radiology, School of Dentistry, Guilan University of Medical Sciences, Rasht, Iran \\ ${ }^{4}$ Rajaie Cardiovascular, Medical and Research Center, Iran University of Medical Sciences, Tehran, Iran \\ *Corresponding author: Fateme Azimi, Department of Oral and Maxillofacial Radiology, School of Dentistry, Guilan University of Medical Sciences, P.O. BOX: 4194173774, Rasht, Iran. \\ Tel: +98-13-33486406, Fax: +98-13-33486423, E-mail: fateme.azimi.dmfr@gmail.com
}

Received: November 7, 2013; Revised: February 10, 2014; Accepted: August 31, 2014

\begin{abstract}
Background: In orthodontic science, diagnosis of facial skeletal type (class I, II, and III) is essential to make the correct treatment plan that is usually expensive and complicated. Sometimes results from analysis of lateral cephalometry radiographies are not enough to discriminate facial skeletal types. In this situation, knowledge about the relationship between the shape and size of the sella turcica and the type of facial skeletal class can help to make a more definitive decision for treatment plan.

Objectives: The present study was designed to investigate this relationship in patients referred to a dental school in Iran.

Patients and Methods: In this descriptive-analytical study, cephalometric radiographies of 90 candidates for orthodontic treatment (44 females and 46 males) with an age range of 14 - 26 years and equal distribution in terms of class I, class II, and class III facial skeletal classification were selected. The shape, length, diameter, and depth of the sella turcica were determined on the radiographs. Linear dimensions were assessed by one-way analysis of variance while the correlation between the dimensions and age was investigated using Pearson's correlation coefficient.

Results: Sella turcica had normal morphology in $24.4 \%$ of the patients while irregularity (notching) in the posterior part of the dorsum sella was observed in $15.6 \%$, double contour of sellar floor in $5.6 \%$, sella turcica bridge in $23.3 \%$, oblique anterior wall in $20 \%$ and pyramidal shape of the dorsum sella in $11.1 \%$ of the subjects. In total, $46.7 \%$ of class I patients had a normal shape of sella turcica, $23.3 \%$ of class II patients had an oblique anterior wall and a pyramidal shape of the dorsum sella, and $43.3 \%$ of class III individuals had sella turcica bridge (the greatest values). Sella turcica length was significantly greater in class III patients compared to class II and class I $(\mathrm{P}<0.0001)$. However, depth and diameter of sella turcica were similar in class I, class II, and class III patients. Furthermore, age was significantly correlated to the diameter of sella turcica as greater diameters were observed in older ages $(\mathrm{P}<0.04)$.

Conclusion: A significant relationship exists between the type of facial skeletal classification and the shape of the sella turcica; as in class III patients, sella turcica bridge was reported with a higher frequency. Also, sella turcica had a significantly higher length in these patients than in those with class I and class II facial skeletal types.
\end{abstract}

Keywords: Sella Turcica; Skeletal; Shape

\section{Background}

For diagnosis of facial skeletal type and assessment of orthodontic treatments, several points in the craniofacial region of patients are used as reference points in tracing lateral cephalometry radiographs. Sella turcica is among the most important orthodontic landmarks. Sella point is of special significance due to its role as the central reference point in the assessment of cranial morphology and intermaxillary relations (1).

The pituitary gland is located in the sella turcica. Thus, various pathologies of this gland can change the shape and size of sella turcica. Some patients with an abnormal sella turcica are suffering from several underlying diseases, intrasellar pituitary primary tumors, hypopituitarism, or syndromes like Williams or Sheehan's syndrome
$(2,3)$. This issue further emphasizes the significance of diagnosis of patients suffering from these conditions by noticing the abnormal shape of the sella turcica (4-6).

Significant personal and ethnic differences have been reported in linear and angular cephalometric measurements of sella turcica in previous studies (7). Therefore, further investigations are required to obtain more data on this subject. Data regarding the morphologic standards of people are necessary in all communities. The morphology of sella turcica is different from person to person. Thus, gaining knowledge in this regard will be a great help in detecting abnormalities in this anatomic area (7).

Since dentists and orthodontists frequently order and

Copyright (C) 2015, Tehran University of Medical Sciences and Iranian Society of Radiology. This is an open-access article distributed under the terms of the Creative Commons Attribution-NonCommercial 4.0 International License (http://creativecommons.org/licenses/by-nc/4.0/) which permits copy and redistribute the material just in noncommercial usages, provided the original work is properly cited. 
evaluate cephalometric radiographies, by learning and knowing the normal variations of the sella turcica, they will be able to recognize abnormalities of this area if there is change in these normal variations, even before the appearance of clinical manifestations $(6,8)$.

At present, determining the morphology of the human craniofacial region is the focus of attention for researchers in various fields of study i.e. radiology and orthodontics (9). A recent theory, still under investigation, is the correlation of shape and size of sella turcica with class I, class II, or class III facial skeletal types. In orthodontic science, facial skeletal type is classified into three types (class I, II, and III) based on the anteroposterior relationship of the maxilla and the mandible (upper and lower jaws). Resolution of this issue can help the diagnosis and treatment of patients who are candidates for orthodontic treatment. Necessary orthodontic treatments for facial skeletal class I, II, and III types are completely different. Before beginning treatments that are usually expensive and complicated it is important to determine the definite type of skeletal relationship between the jaws. Sometimes, measurements resulting from lateral cephalometric analysis may provide borderline findings and it is not possible to differentiate between different skeletal types, especially class I and class III. So, making definitive decision for the treatment plan is difficult. In this situation, attention to the variation in the shape of the sella turcica on the existing cephalometric radiograph that was analyzed before for cephalometric measurements helps to determine if the skeletal relationship is progressing to class III or class I.

\section{Objectives}

Studies conducted on this subject have been performed in other countries and on people with different ethnicities. Considering the importance of this issue, this study was conducted on a small sample size representative of our country's population with the aim of determining the correlation between the shape and size of the sella turcica and the classification of facial skeletal type.

\section{Patients and Methods}

This retrospective descriptive analytical study was conducted on 90 patients aged 14 - 26 years who presented to an Iranian dental school clinic for orthodontic treatment during 2011 - 2012 and their cephalometric radiographies were present in their dental record file. This sample was obtained out of a total of 1210 records in the mentioned age group using simple random sampling method. The sequence was generated by Microsoft Excel $₫$ software.

This age range was selected on purpose because in the post-pubertal age group (ages $>14$ years) no considerable change occurs in the shape or size of the sella turcica (10). Based on patients' records, those with a history of maxillofacial trauma, syndromic or non- syndromic craniofacial clefts and history of maxillofacial surgery, en- docrine disorders, patients using hormonal medications or corticosteroids and those suffering from syndromes with craniofacial abnormalities were excluded from the study. Cephalometric radiographies were obtained from 90 patients (44 females and 46 males) using PM2002 CC Proline Panoramic Ceph X ray Machine (Planmeca, USA, Inc). Radiographs were taken by an expert, trained technician in a standard position with a specific device and a cephalostat with a fixed magnification factor. Only the radiographs showing a crystal clear image of the sella turcica were selected for interpretation and analysis. Selected radiographs were then classified according to different facial skeletal patterns and gender (30 class I, 30 class II and 30 class III skeletal type with equal distribution of males and females). Radiographs were divided into different classes based on ANB angle value (SNA and $\mathrm{SNB}$ ) (class I: ANB angle equal to $\pm 2^{\circ}$, class II: ANB angle $>4^{\circ}$, class III: ANB angle $<0^{\circ}$, ANB angle range: 0 to $4^{\circ}$ ) (11). Facial skeletal pattern is classified into three types, based on the anteroposterior relationship of the maxilla and the mandible (class I, II, and III). These three types are explained by ANB angle based on steiner analysis. "A", "N" and "B" points are detectable on lateral cephalometric radiographies and present $A$ : the deepest point of the anterior border of the maxillary alveolar ridge concavity. $\mathrm{N}$ : nasion (frontonasal suture), and $\mathrm{B}$ : the deepest point of the concavity of the anterior border of the mandible (Figure 1) (10).

In order to overcome limitations of ANB angle, Wits analysis was used (11). Considering the fact that understudy patients were candidates for orthodontic treatment in the dental school, final confirmation of the facial skeletal classification was done based on their records and after the approval of their assigned orthodontist.

The sella turcica region was traced on each lateral cephalometric radiography on thin acetate paper under ideal lighting. Then, different parts of the sella turcica including tuberculum sella, floor of sella turcica, dorsum sella and anterior and posterior clinoid processes were all traced (Figure 2).

The shape of the sella turcica was categorized into six groups including normal morphology and its anatomical variations namely oblique anterior wall, sella turcica bridging, double contour of sellar floor, irregularity (notching) in the posterior part of the dorsum sella and pyramidal shape of the dorsum sella (12) (Figure 3). The shape of the sella turcica in each radiograph was recorded after approval and reaching a consensus between three radiologist observers.

For linear measurements, a standard method introduced by Silverman (1957) (13) and Kisling (1966) (14) was used. The length of the sella turcica was measured from the tuberculum sella to the tip of the dorsum sella. The depth of the sella turcica was calculated by drawing a line perpendicular to the line passing through the deepest point on the floor of sella. The anteroposterior diameter of the sella turcica was measured by drawing a line from 
Valizadeh $S$ et al.

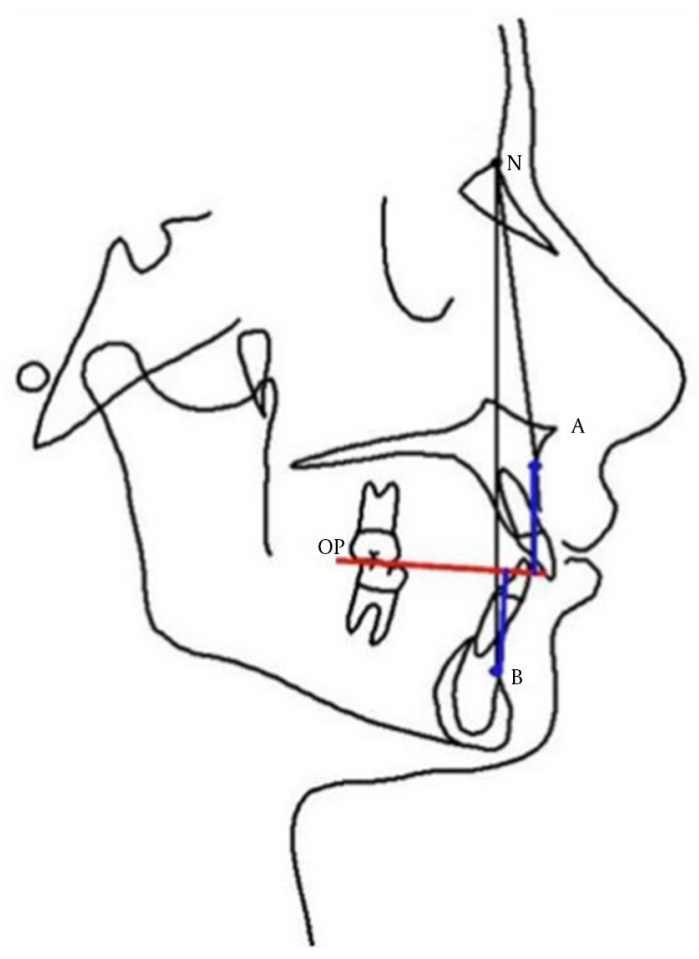

Figure 1. Anteroposterior relationship of the maxilla and the mandible based on steiner analysis. A, the deepest point of the anterior border of the maxillary alveolar ridge concavity; N, nasion (frontonasal suture); B, the deepest point of the concavity of the anterior border of the mandible; Class I: the mean reading for ANB angle is 2 degrees. Class II: a reading greater than 2 degrees for ANB angle indicates a class II skeletal tendency and angles less than 2 degrees and readings less than zero (e. g. -1 degree, -2 degrees, -3 degrees) indicate that the mandible is located ahead of the maxilla suggesting a class III skeletal relationship.

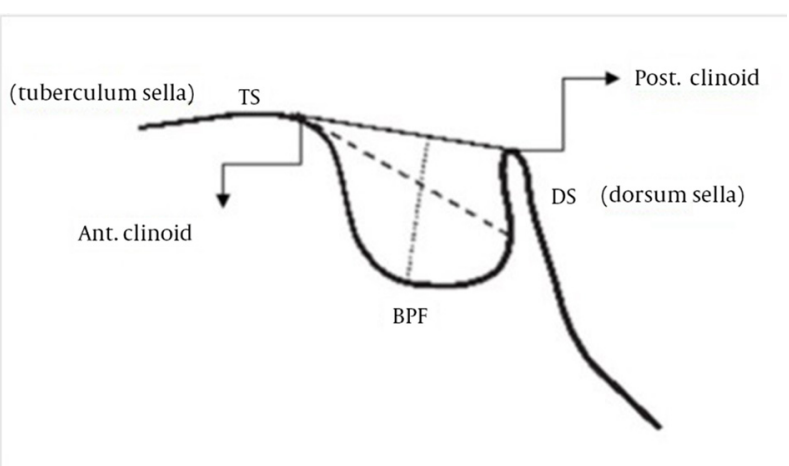

Figure 2. Normal morphology of sella turcica along with reference lines for measuring the size of sella. Abbreviations: TS, tuberculum sella; DS, dorsum sella; BPF, base of pituitary fossa; Explanations: black line, length of sella; dash line, sella diameter; dotted line, depth of sella

the tuberculum sella to the most distant point on the posterior inner wall of the fossa (Figure 2).
All measurements were done by a single rater, using a ruler by considering a fixed magnification factor of 1.1 for all radiographs. Intra-observer reliability was assessed on 15 random images that were chosen again to determine the shape of the sella turcica. The agreement between two measurements was evaluated using weighted kappa statistics. Data were analyzed using SPSS ver. 15 for Windows (SPSS Inc., Chicago. Illinois).

Data were presented as Mean \pm SD for interval and count (\%) for categorical variables. One way ANOVA was employed to compare the dimensions of sella turcica in its different shapes and facial skeletal classes. Pearson's chi square and Fisher's exact tests were also used. The effect of age, sex, and facial skeletal type on the dimensions of sella turcica was evaluated using multiple linear regression models. P value lower than 0.05 was considered as statistically significant.

\section{Results}

Evaluation of the normal variants of the anatomical shapes of the sella turcica in this study revealed that the shape of sella turcica was normal in $22(24.4 \%)$ subjects, $14(15,6 \%)$ had irregularity (notching) in the posterior part of the dorsum sella, five (5.6\%) had double contour of sellar floor, 21 (23.3\%) had sella turcica bridging, 18 (20\%) had oblique anterior wall and 10 cases (11.1\%) had pyramidal shape of the dorsum sella.

Associations between facial skeletal types, patients' demographic data and characteristics of sella turcica are presented in Table 1. The majority of class I cases showed normal morphology of sella turcica $(48.3 \%)$, most class II patients had oblique anterior wall and pyramidal shape of the dorsum sella with a prevalence of $25.8 \%$ and $22.6 \%$ respectively and the majority of patients with class III skeletal type, had sella turcica bridging (43.3\%). No associations between age, sex, and anatomical measures and facial skeletal classes were observed (Table 1).

In the normal shape of sella, the mean length was 9.04 $\mathrm{mm}$. This measure was $8.78,8.64,8.22,9.43$, and $8.82 \mathrm{~mm}$ in variations like irregularity (notching) in the posterior part of the dorsum sella, double contour of sellar floor, sella turcica bridging, oblique anterior wall, and pyramidal shape of the dorsum sella, respectively. No statistically significant difference was detected in the length of sella turcica between its normal variants $(\mathrm{P}=0.254)$.

In the normal shape of sella, the mean depth was 8.03 $\mathrm{mm}$ while this measure was 7.84, 6.84, 8.06, 8.1 and 7.74 $\mathrm{mm}$ in variations like irregularity (notching) in the posterior part of the dorsum sella, double contour of sellar floor, sella turcica bridging, oblique anterior wall and pyramidal shape of the dorsum sella, respectively. No statistically significant difference was detected in the depth of sella turcica between its normal variants $(\mathrm{P}=0.184)$.

However, a statistically significant difference was detected in the diameter of sella turcica between its various 

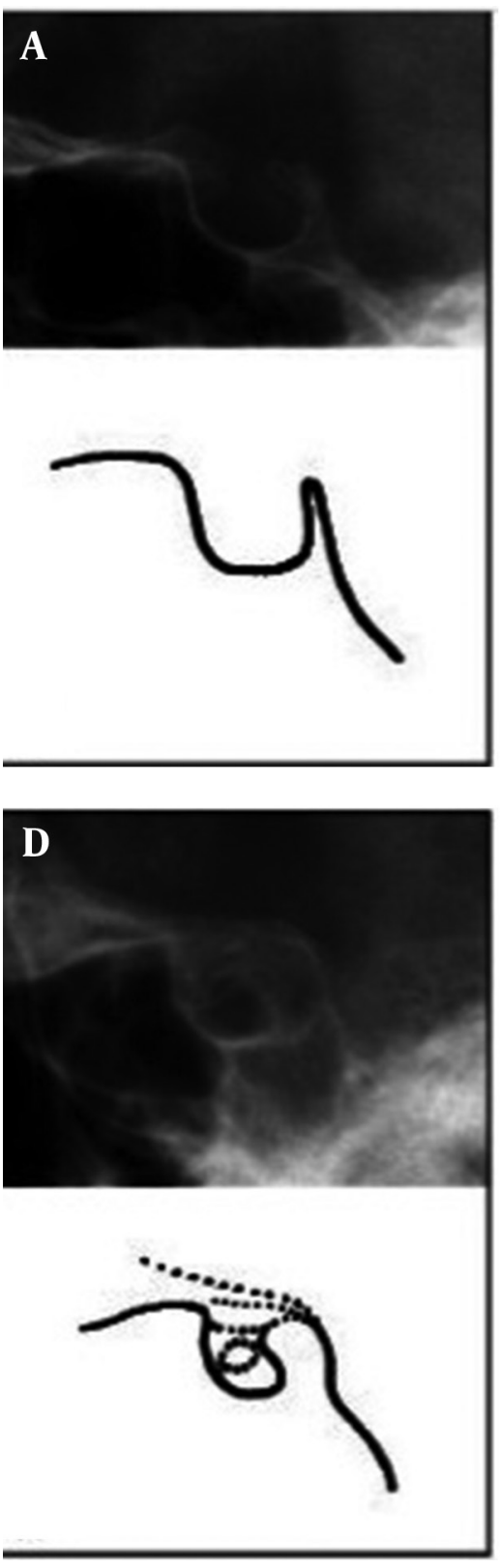
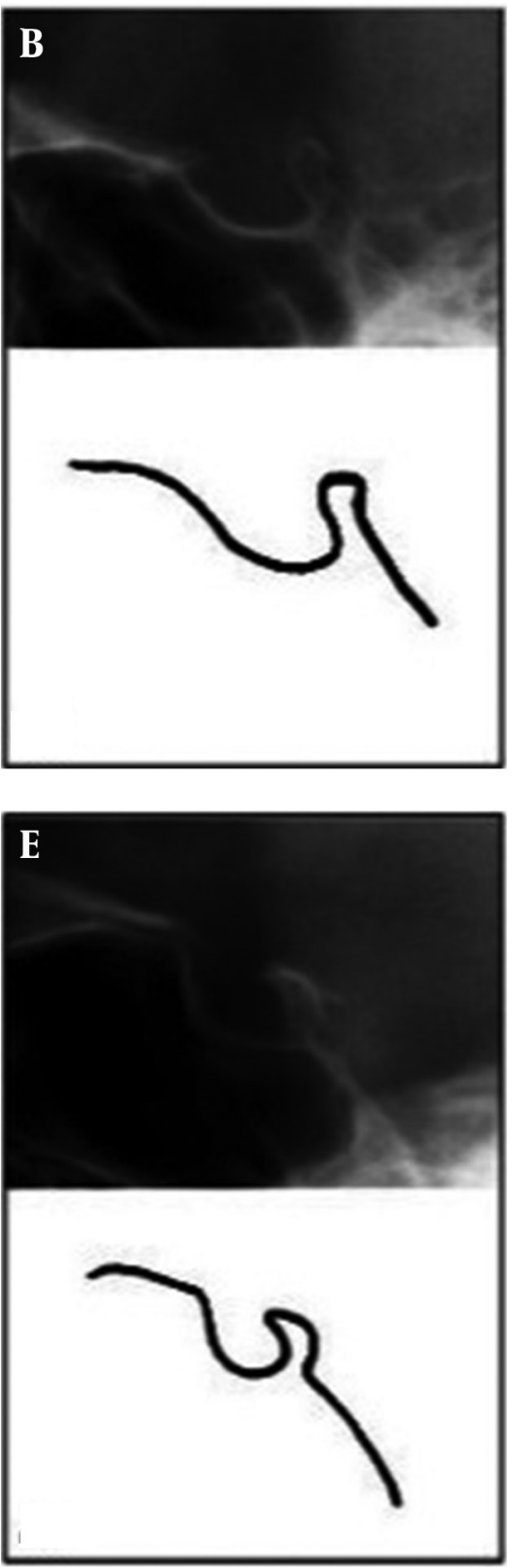
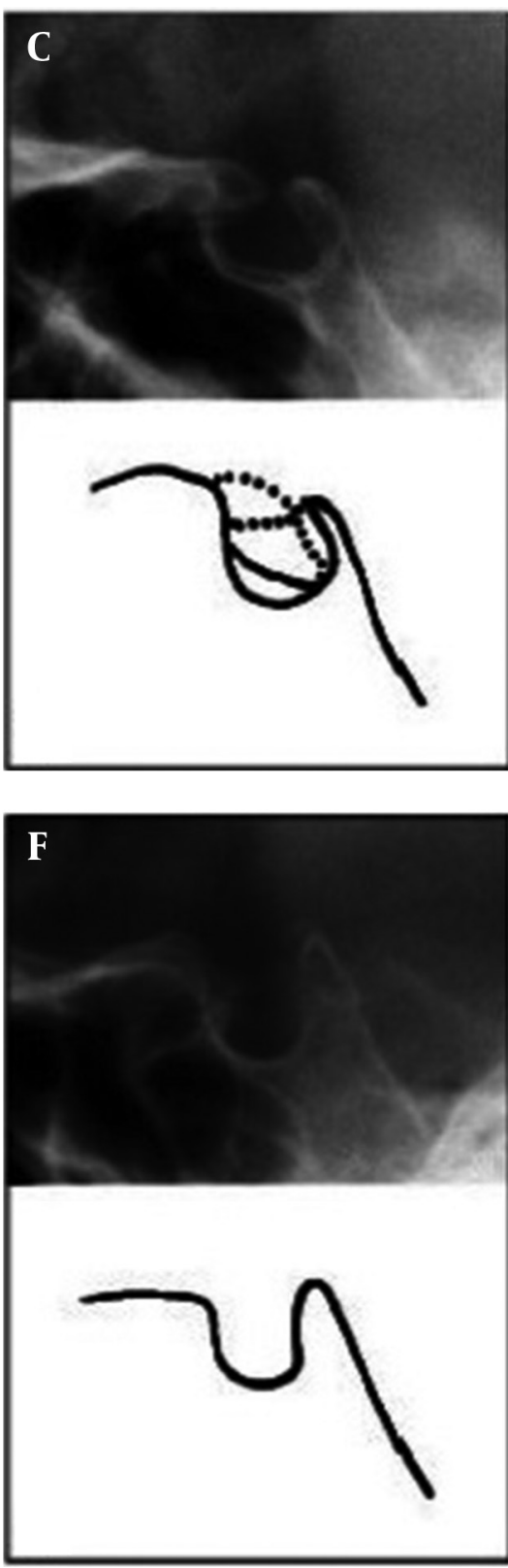

Figure 3. Morphologic classification of sella turcica. A, Normal sella turcica; B, Oblique anterior wall, C, Double contour of sellar floor, D, Sella turcica bridge, E, Irregularity (notching) in the posterior part of the dorsum sella and F, Pyramidal shape of dorsum sella.

forms $(P=0.02)$. The mean diameter of sella turcica in its normal shape and different variations is demonstrated in Table 2. As observed, this diameter was slightly greater in the oblique anterior wall, irregularity (notching) in the posterior part of the dorsum sella and normal shape of sella turcica compared to other variations. On the other hand, results of the present study demonstrated that patients' age had a direct and significant correlation with the diameter of sella turcica $(\mathrm{P}<0.04)$ and by advanced age, the diameter of the sella turcica significantly increased. However, the correlation of age with length (P
$=0.166)$ and depth $(P=0.866)$ of sella turcica was weak and insignificant.

To investigate the associations between anatomical measures of sella turcica (length, depth and diameter) and facial skeletal types, adjusted for patients' age and sex, multiple linear regression models were applied. The results showed that after the adjustment, the associations between all the factors were not statistically significant (Table 3). Kappa (for measuring intra-observer reliability) was 0.74 , which represent a good agreement and therefore, the reliability was acceptable. 
Valizadeh $S$ et al.

\begin{tabular}{|c|c|c|c|c|}
\hline \multirow[t]{2}{*}{ Variables } & \multicolumn{3}{|c|}{ Facial Skeletal Classification } & \multirow[t]{2}{*}{ P Value } \\
\hline & Class I $(\mathbf{n}=\mathbf{2 9})$ & Class II $(\mathbf{n}=\mathbf{3 1})$ & Class III $(\mathbf{n}=\mathbf{3 0})$ & \\
\hline \multicolumn{5}{|l|}{ Demographic Characteristics } \\
\hline Age, $y$ & $17.03 \pm 4.16$ & $16.1 \pm 3.89$ & $16.5 \pm 3.63$ & 0.491 \\
\hline Gender, F/M & $10 / 19$ & $19 / 12$ & $17 / 13$ & 0.088 \\
\hline \multicolumn{5}{|l|}{ Characteristics of Sella Turcica } \\
\hline Length, $\mathrm{mm}$ & $10.43 \pm 1.48$ & $10.34 \pm 2.1$ & $9.52 \pm 2.23$ & 0.124 \\
\hline Depth, mm & $8.83 \pm 1.21$ & $8.53 \pm 1.47$ & $8.45 \pm 1.39$ & 0.420 \\
\hline Diameter, mm & $13.02 \pm 1.57$ & $12.06 \pm 2.4$ & $12.12 \pm 1.9$ & 0.261 \\
\hline Shape & & & & 0.007 \\
\hline Normal $^{\mathrm{a}}$ & $14(48.3)$ & $5(16.1)$ & $3(10)$ & \\
\hline $\begin{array}{l}\text { Irregularity (notching) in the posterior part of the } \\
\text { dorsum sella a }\end{array}$ & $5(17.2)$ & $5(16.1)$ & $4(13.3)$ & \\
\hline Double contour of sellar floor ${ }^{a}$ & $1(3.4)$ & $2(6.5)$ & $2(6.7)$ & \\
\hline Sella turcica bridging ${ }^{a}$ & $4(13.8)$ & $4(12.9)$ & $13(43.3)$ & \\
\hline Oblique anterior wall ${ }^{\mathrm{a}}$ & $4(13.8)$ & $8(25.8)$ & $6(20)$ & \\
\hline Pyramidal shape of the dorsum sella ${ }^{a}$ & $1(3.4)$ & $7(22.6)$ & $2(6.7)$ & \\
\hline
\end{tabular}

a values are present as No. (\%).

Table 2. Description of Sella Turcica Diameter in Patients in Different Sub-Groups of Shape

\begin{tabular}{lccc}
\hline Variables & Mean & Standard Deviation & Standard Error \\
\hline Normal & 11.69 & 1.56 & 0.28 \\
\hline $\begin{array}{l}\text { Irregularity (notching) in the posterior part of the } \\
\text { dorsum sella }\end{array}$ & 11.89 & 1.52 & 0.41 \\
Double contour of sellar floor & 9.9 & 0.64 & 0.28 \\
Sella turcica bridging & 10.39 & 1.91 & 0.58 \\
Oblique anterior wall & 12.22 & 1.86 & 0.44 \\
Pyramidal shape of the dorsum sella & 10.75 & 2.43 & 0.77 \\
\hline
\end{tabular}

Table 3. Multiple Linear Regression Models to Show the Associations Between Facial Skeletal Class and Anatomical Characteristics of Sella Turcica, Adjusted for Age and Sex

\begin{tabular}{|c|c|c|c|c|c|c|}
\hline \multirow[t]{2}{*}{ Variables } & \multicolumn{2}{|c|}{ Length $\left(r^{2}=0.09\right)$} & \multicolumn{2}{|c|}{ Depth $\left(r^{2}=0.02\right)$} & \multicolumn{2}{|c|}{$\operatorname{Diameter}\left(\mathbf{r}^{2}=\mathbf{0 . 0 9}\right)$} \\
\hline & $\beta^{a}$ & PValue & $\beta^{a}$ & P Value & $\beta^{a}$ & P Value \\
\hline Facial skeletal Class & -0.349 & 0.178 & -0.168 & 0.358 & -0.336 & 0.201 \\
\hline Age & 0.098 & 0.075 & 0.018 & 0.645 & 0.104 & 0.061 \\
\hline Gender & 0.772 & 0.072 & 0.138 & 0.647 & 0.765 & 0.078 \\
\hline
\end{tabular}

a $\beta$ : coefficient of variables in the regression model.

\section{Discussion}

Based on the present study results, the shape of the sella turcica was normal in $24.4 \%$ of the subjects. Other variations were observed in $75.6 \%$ of the cases. However, Alkofide in his study evaluated the shape and size of sella turcica in patients with facial class I, class II, and class III skeletal classifications and reported normal morphology of sella turcica in $67 \%$ of the cases. The remaining $33 \%$ presented with variations of sella turcica morphologies (10). In a study conducted by Mahmood Shah, the normal morphology was also seen in approximately $66 \%$ of the subjects (15).

The frequency of the normal morphology of sella turcica in the present study was lower than the rates reported by Alkofide, Axelsson and Mahmood Shah et al. This dif- 
ference can be due to the different ethnicity of the study samples $(10,12,15)$. Occurrence of sella turcica bridging is also possible in healthy individuals. Incidence of this normal variation has reported to be within the range of 5.5 to $22 \%(12,16)$. However, its prevalence has reported to be higher in patients suffering from craniofacial disorders (17). In the present study, sella turcica bridge was present in $23.3 \%$ of the subjects. In the study conducted by Alkofide, $1.1 \%$ of patients had sella turcica bridge (10). In the study carried out by Becktor et al. sella turcica bridge was reported in $18.6 \%$ of all patients with severe craniofacial disorders (17). Based on the results of a study performed by Axelsson et al. on morphology and size of sella turcica in patients suffering from Williams's syndrome, sella turcica bridge was seen in $13 \%$ of the patients (12). Leonardi et al. reported that the prevalence of sella turcica bridge is higher in adolescents with dental anomalies (18). AbdelKader evaluated the prevalence of sella turcica bridge in patients who were candidates for orthodontic treatment and reported its prevalence to be $3.74 \%$ (19). Based on the present study results, sella turcica bridge was present in $13.3 \%$ of skeletal class I, $13.3 \%$ of class II and $43.3 \%$ of skeletal class III patients. These rates were all greater than the results of previous studies.

Meyer-Marcotty et al. reported the frequency of sella turcica bridging to be greater in skeletal class III patients compared to class I (16.8\% versus $9.4 \%)$. These findings are in accordance with our study results (20). Based on the present study results, no statistically significant difference was detected between males and females in length, depth or diameter of the sella turcica. Similar results were also reported by Alkofide (10) and Mahmood Shah (15).

However, patients' age had a direct and statistically significant correlation with the diameter of sella turcica and by advanced age, the diameter of the sella turcica constantly increased. However, no such correlation existed between the length and depth of the sella turcica with age. In the study conducted by Alkofide, size of sella turcica was larger in the older age group (10). Furthermore, Preston found a close correlation between the pituitary fossa size and age (21). Choi et al. also reported that linear dimensions of sella turcica had positive inclination till the age of 25 (9) although no significant increase was reported in the size of sella turcica after the age of 26 (10). Limited studies have been conducted on the effect of different facial skeletal patterns on the size of sella turcica. In the present study, only the length of sella turcica in class III patients was significantly greater than that in class I and class II patients and the depth and diameter of sella turcica in class I, class II, and class III patients were relatively the same. In contrast to our study results, Preston could not find a significant association between facial types and the mean size of pituitary fossa $(15,21)$. However, Alkofide in his study found significant associations between different facial skeletal classifications and linear dimensions of the sella turcica (significant differ- ences in sella turcica diameter between class II and class III patients) (10).

In the present study, manual tracing was used for calculation of the length, depth and diameter of the sella turcica. Although in some studies the digital method was used to measure these factors, the manual technique has accuracy similar to that of digital technique in this regard (12). Thus, considering its affordability, the manual technique was used. It seems that further investigations in several centers with larger sample sizes can increase the accuracy of the obtained data and standards.

Based on the present study results, a significant correlation was detected between facial skeletal type and the shape of sella turcica as sella turcica bridging had a higher frequency in class III facial skeletal type patients. Also, the length of sella turcica in these patients was significantly higher compared to that in class I and class II patients. Additionally, a direct correlation existed between patients' age and the diameter of sella turcica and by advanced age, the diameter of the sella turcica significantly increased.

\section{Authors' Contributions}

Study concept and design: Solmaz Valizadeh; 2- Acquisition of data: Shahrzad Shahbeig; 3- Analysis and interpretation of data: Fateme Azimi; 4- Drafting of the manuscript: Sudeh Mohseni; 5- Critical revision of the manuscript for important intellectual content: Solmaz Valizadeh; 6- Statistical analysis: Hooman Bakhshandeh; 7- Administrative, technical, and material support: Shahrzad Shahbeig, Sudeh Mohseni; 8- Study supervision: Solmaz Valizadeh.

\section{Funding/support}

This study was supported by Shahid Beheshti University of Medical Sciences.

\section{References}

1. Pisaneschi M, Kapoor G. Imaging the sella and parasellar region Neuroimaging Clin N Am. 2005;15(1):203-19.

2. Elster AD. Imaging of the sella: anatomy and pathology. Semin Ultrasound CT MR. 1993;14(3):182-94.

3. Andredaki M, Koumantanou A, Dorotheou D, Halazonetis DJ. A cephalometric morphometric study of the sella turcica. EurJ Orthod. 2007;29(5):449-56.

4. Weisberg LA, Zimmerman EA, Frantz AG. Diagnosis and evaluation of patients with an enlarged sella turcica. Am J Med. 1976;61(5):590-6.

5. Friedland B, Meazzini MC. Incidental finding of an enlarged sella turcica on a lateral cephalogram. Am J Orthod Dentofacial Orthop. 1996;110(5):508-12.

6. Kjaer I, Hjalgrim H, Russell BG. Cranial and hand skeleton in fragile X syndrome. Am J Med Genet. 2001;100(2):156-61.

7. Zagga AD, Ahmed H, Tadros AA, Saidu SA. Description of the normal variants of the anatomical shapes of the sella turcica using plain radiographs: experience from Sokoto, Northwestern Nigeria. Ann Afr Med. 2008;7(2):77-81.

8. Alkofide EA. Sella turcica morphology and dimensions in cleft subjects. Cleft Palate Craniofac J. 2008;45(6):647-53.

9. Choi WJ, Hwang EH, Lee SR. The study of shape and size of nor- 
mal sella turcica in cephalometric radiographs. Korean J Oral and Maxillofacial Radiol. 2001;31(1):43-9.

10. Alkofide EA. The shape and size of the sella turcica in skeletal Class I, Class II, and Class III Saudi subjects. Eur J Orthod. 2007;29(5):457-63.

11. Proffit WR, Sarver DM, Ackerman JL. Orthodontic Diagnosis: The Problem-Oriented Approach. In: Proffit WR, Fields HW, Sarver DM, editors. Contemporary Orthodontics. St Louis: Elsevier; 2013. pp. 150-214.

12. Axelsson S, Storhaug K, Kjaer I. Post-natal size and morphology of the sella turcica. Longitudinal cephalometric standards for Norwegians between 6 and 21 years of age. Eur J Orthod. 2004;26(6):597-604.

13. Silverman FN. Roentgen standards for size of the pituitary fossa from infancy through adolescence. AmJ Roentgen. 1957;78(3):451460.

14. Kisling E. A comparative roentgencephalometric study in adult males 1966. Copenhagen: Munksgaard; 1966.

15. Mahmood Shah A, Bashir U, Tasleem I. The shape and size of the sella turcica in skeletal class I, II \& III in patients presenting at Islamic International Dental Hospital, Islamabad. Pakistan Oral and Dental Journal. 2011;31(1):104-10.

16. Kantor ML, Norton LA. Normal radiographic anatomy and common anomalies seen in cephalometric films. Am JOrthod Dentofacial Orthop. 1987;91(5):414-26.

17. Becktor JP, Einersen S, Kjaer I. A sella turcica bridge in subjects with severe craniofacial deviations. Eur J Orthod. 2000;22(1):69-74.

18. Leonardi R, Barbato E, Vichi M, Caltabiano M. A sella turcica bridge in subjects with dental anomalies. Eur J Orthod. 2006;28(6):580-5.

19. Abdel-Kader HM. Sella turcica bridges in orthodontic and orthognathic surgery patients. A retrospective cephalometric study. Aust Orthod J. 2007;23(1):30-5.

20. Meyer-Marcotty P, Reuther T, Stellzig-Eisenhauer A. Bridging of the sella turcica in skeletal Class III subjects. Eur J Orthod. 2010;32(2):148-53.

21. Preston CB. Pituitary fossa size and facial type. Am J Orthod. 1979;75(3):259-63. 